\title{
Les cours d'eau récepteurs de rejets de stations d'épuration : le cas de la Mauldre (Île-de-France), un milieu sous très haute pression
}

\section{Receiving fresh water from domestic wastewater treatment plant outlet: a case study for Mauldre (Ile-de-France), a medium under very high pressure}

\section{Figuet et J. P. Frangi}

Volume 13, numéro 2, 2000

URI : https://id.erudit.org/iderudit/705385ar

DOI : https://doi.org/10.7202/705385ar

Aller au sommaire du numéro

\section{Éditeur(s)}

Université du Québec - INRS-Eau, Terre et Environnement (INRS-ETE)

\section{ISSN}

0992-7158 (imprimé)

1718-8598 (numérique)

Découvrir la revue

Citer cet article

Figuet, C. \& Frangi, J. P. (2000). Les cours d'eau récepteurs de rejets de stations d'épuration : le cas de la Mauldre (Île-de-France), un milieu sous très haute pression. Revue des sciences de l'eau / Journal of Water Science, 13(2), 119-138. https://doi.org/10.7202/705385ar

\section{Résumé de l'article}

L'étude des impacts des rejets des stations d'épuration communales sur les cours d'eau récepteurs est généralement conduite selon des méthodes d'analyses ponctuelles en rivière. Mais ces méthodes ne donnent pas des résultats satisfaisants car elles ne tiennent pas compte à la fois de la qualité des cours d'eau et des rejets, et nient le continuum de la rivière en analysant des points successifs. D'autres méthodes, globales, tiennent compte des flux en rivière et en station et fournissent des résultats plus représentatifs des phénomènes qui se produisent en rivière. Une étude de cas a été menée sur la Mauldre et ses affluents. Deux méthodes de calcul d'impact ponctuel ont été utilisées, le ratio de dilution et le taux de pollution. Un bilan des flux en rivière et en station a été mis au point pour l'analyse globale. Celle-ci a été complétée par un bilan global des impacts, issu d'une approche systémique qui élargit le périmètre des investigations. Cette dernière méthode demande l'acquisition de peu de données en comparaison des autres. Elle est également plus intégratrice, replace le problème posé dans un contexte plus vaste et permet ainsi d'y apporter une réponse intéressante. 


\title{
Les cours d'eau récepteurs de rejets de stations d'épuration : le cas de la Mauldre (lle-de-France), un milieu sous très haute pression
}

\author{
Receiving fresh water from domestic wastewater \\ treatment plant outlet: a case study for Mauldre \\ (lle-de-France), a medium under very high pressure
}

C. FIGUET ${ }^{1,2}$, J.-P. FRANGI ${ }^{1, *}$

Reçu le 28 mars 1999, accepté le 27 janvier 2000**.

\section{SUMMARY}

\begin{abstract}
Rivers are sensitive and vulnerable ecosystems that may be disturbed or even distorted by human activities. The most important consequences are changes in quality or quantity of physical, chemical or biological water characteristics. The impacts of human activities are sometimes immense and last a very long time: extensive engineering projects, illegal polluted water outfalls, direct storm water outfalls, combined sewer overflows, and effluents from wastewater treatment plants treating domestic and connected industries might be listed, but this list is clearly not complete. With particular reference to wastewater treatment plant effluents, it is clear that such plants cannot get rid of the whole amount of pollutant loads from domestic and connected activities. The local pollutant pressure generated by wastewater treatment plants outfalls leads to physical, chemical and bacteriological pollution of the receiving fresh waters, potentially with great impact on the river system downstream.
\end{abstract}

Studies downstream from wastewater treatment plant outfalls allow one to identify pollutant zones or pollution risks, and secondly provide a better understanding of the phenomena occurring in the receiving waters. Another goal of this work was to foster better environmental management practices; the conclusions are dedicated to the staff in charge of the SAGE "Schema d'Aménagement et de Gestion des Eaux" of the Mauldre river.

A case study was undertaken on the river Mauldre and its tributaries, an environment under very high pollution stress. The river Mauldre is a small river in the Seine catchment. Its main stream flows from South to North and joins the Seine at Epône; the hydrological catchment area is $410 \mathrm{~km}^{2}$. The ecological and biological functioning of the river Mauldre has been highly affected by human activities for a very long time. The East part of the

1. Laboratoire environnement et développement, CP 7071, Université Paris VII-Denis Diderot, 2 pl. Jussieu, 75251 Paris cedex, France.

2. Agence de l'eau Seine-Normandie, 51 rue S. Allende, 92028 Nanterre cedex, France.

* Correspondance. E-mail : frangi@ccr.jussieu.fr

** Les commentaires seront reçus jusqu'au 15 mai 2001. 
Mauldre catchment is mainly an urban and industrial area covered by big agglomerations such as Élancourt, Plaisir or Versailles, which are all located upstream from the right side tributaries. The West part of the catchment has a more agricultural aspect, with small villages and wide prairies or fields. To get rid of the water pollution loads from domestic and connected activities, twenty-three wastewater treatment plants have been built since 1950 , with a total theoretical capacity of $\mathbf{4 4 0} 999$ population-equivalents. These stations treat most of the total pollution loads (88\% BOD removal), but for phosphorus and nitrogen removal is less efficient $(60 \%$ and $30 \%$, respectively), since most of these stations are old and do not carry out very thorough treatments. The quality of the rivers is thus degraded by the anthropogenic pressure that is exerted on this catchment area.

Impact studies on wastewater effluents are generally performed according to methods involving specific point analyses. We used two of these calculation methods: the ratio of dilution and the rate of pollution. The first method calculates the river's dilution power according to the relation between the rate of low flow in the river and the average annual flow of the effluent from the upstream wastewater treatment plant (dimensionless). The second method, the rate of pollution or "Trabuc index", determines the wastewater treatment plant contribution to downstream water quality according to the formula: quantity of pollutant discharged in the treatment plant effluent/rate of low flow in the river (units $=\mathbf{m g} \cdot \mathrm{L}^{-1}$ ). The application of the two calculation methods application does not give satisfactory results because they do not take into account the quality of the rivers or the quality of the wastewater treatment plant effluents. Furthermore, they ignore the river continuum by analysing successive point events. Both methods provide similar results: impacts of wastewater treatment plant effluents on the river are decreasing downstream. These results are discussed and compared with in situ river pollution measurements made especially for this work. The measurements show an increasing pollutant impact downstream, which contradicts the two calculation methods results used at the beginning of this study.

Therefore we investigated the use of global methods, which take into account more of the river's phenomena, using our measurement results. First we developed a more satisfactory assessment of pollutant loads in the river and in the wastewater treatment effluents. The results of this revised calculation method are not far from our observed river data. They take also into account the quality of the wastewater treatment plant effluents.

This method consists only of a one-dimensional basic model and gives a global description of river behaviour. This analysis was enhanced by a global assessment of the impacts, resulting from a systems approach. We refers our analysis to Joël de Rosnay's work, "Le macroscope" (DE ROSNAY, 1975). This approach involves the identification of structural and functional aspects of the studied system, using a new vocabulary: we talk of elements (river, water chemical elements, wastewater treatment plant, population, industries), frontiers (of the atmosphere, the hydrological or hydrogeological catchment) and internal or external links. The usual framework widens and the central question of this paper is considered in a broader context, the whole catchment and its components, furnishing brief answers. This method provides many points of view on our studied subject, and highlights new components and new relations among them. At the end of the study we discuss a very interesting relation between inhabitants of the catchment and low flow, which find expression in the population stress index. The wastewater treatment plant pressure on the river appears as an initial demographic pressure on the catchment. The last method we used needs fewer data than the others do, but give us a surprisingly accurate and highly significant index of the impact of wastewater treatment plant effluents.

Key-words: river, wastewater treatment plant, polluted water outlet, water pollution, systemic, Mauldre. 


\section{RÉSUMÉ}

L'étude des impacts des rejets des stations d'épuration communales sur les cours d'eau récepteurs est généralement conduite selon des méthodes d'analyses ponctuelles en rivière. Mais ces méthodes ne donnent pas des résultats satisfaisants car elles ne tiennent pas compte à la fois de la qualité des cours d'eau et des rejets, et nient le continuum de la rivière en analysant des points successifs. D'autres méthodes, globales, tiennent compte des flux en rivière et en station et fournissent des résultats plus représentatifs des phénomènes qui se produisent en rivière. Une étude de cas a été menée sur la Mauldre et ses affluents. Deux méthodes de calcul d'impact ponctuel ont été utilisées, le ratio de dilution et le taux de pollution. Un bilan des flux en rivière et en station a été mis au point pour l'analyse globale. Celle-ci a été complétée par un bilan global des impacts, issu d'une approche systémique qui élargit le périmètre des investigations. Cette dernière méthode demande l'acquisition de peu de données en comparaison des autres. Elle est également plus intégratrice, replace le problème posé dans un contexte plus vaste et permet ainsi d'y apporter une réponse intéressante.

Mots clés : cours d'eau, stations d'épuration, rejets, pollution, analyses globales, systémique, Mauldre.

\section{1 - INTRODUCTION ET PRÉSENTATION DU SITE EXPÉRIMENTAL}

Les rivières sont des écosystèmes sensibles et fragiles que les actions humaines perturbent et parfois même détruisent. Les impacts de ces activités sont souvent très forts et perdurent longtemps. C'est le cas des rejets issus des activités domestiques qui aboutissent dans la plupart des cas dans les cours d'eau, après des traitements de dépollution partielle dans les stations d'épuration. La pression exercée par ces stations d'épuration sur les cours d'eau récepteurs engendre une pollution physique, chimique et bactériologique. Elle est le plus souvent étudiée soit à l'aide de modèles physicochimiques de divers types, soit par des ratios de dilution. Dans les deux cas, il s'agit de données physicochimiques qui sont utilisées de manière binaire: des données sur le milieu naturel d'un côté, des données sur les rejets des stations de l'autre. Quelques modèles ont été mis au point sur des grands fleuves : par exemple Kalito (LESOUEF et ANDRE, 1982), Monet (MEYBECK, 1998b), Prose (EVEN, 1995) étudient pour la Seine les interactions entre différentes variables physiques, chimiques et biologiques (modèles unidimensionnels) comme le débit, la concentration en oxygène dissous, la vitesse de minéralisation de l'azote... Mais quand il s'agit d'une petite rivière, ces modèles ne sont plus valides et ils se heurtent vite au manque de données sur les milieux naturels et aux difficultés de calage dues à des débits faibles et à des milieux qui recèlent des caractéristiques très hétérogènes. C'est pourquoi les modèles qui couplent les rejets de stations et les rivières ne donnent pas de résultats très satisfaisants.

Les ratios de dilution des effluents de station d'épuration des eaux sont plus restrictifs que les modèles car ils utilisent moins de paramètres, et analysent ponctuellement les relations entre les rejets des stations d'épuration et les rivières. Ils utilisent des critères quantitatifs comme le fait le ratio Diren (Diren lle-de-France, 1996), ou bien des critères quantitatifs et qualitatifs chimiques 
pour les stations comme l'indice Trabuc (MORIZE, 1984). Extraire ainsi des éléments jugés représentatifs de la réalité observée pour élaborer des indicateurs simplifie très fortement la réalité ainsi que la réponse au problème posé par les relations entre les rejets et les rivières. Le phénomène d'auto-épuration est généralement pris en compte dans l'étude des relations rejets/cours d'eau. Cependant, en raison de débits naturels très faibles et de rejets trop importants en volume et en charge polluante sur la Mauldre, cet aspect a été écarté de la présente étude, car il n'a pas été mis en évidence durant une campagne mensuelle en 1998 et une autre de 24 heures menée en septembre 1998. Afin d'analyser ces relations rejets/rivières, nous présenterons et discuterons plusieurs méthodes. Dans un premier temps nous exposerons deux ratios de dilution que nous confronterons dans un deuxième temps à une méthode de bilan des flux chimiques, à l'aide d'une étude de cas sur la Mauldre et ses affluents (lle-de-France). En effet, ces relations seront replacées à l'échelle du bassin versant et à l'échelle globale par une approche spatiale et systémique.

\section{Présentation du site}

La Mauldre est un petit affluent en rive gauche de la Seine, en aval de l'agglomération parisienne. Son cours principal coule sud-nord sur $50 \mathrm{~km}$, et se jette en Seine au niveau d'Epône. Son bassin versant hydrographique s'étend sur une superficie approximative de $410 \mathrm{~km}^{2}$. Bassin de taille modeste à l'échelle de l'lle-de-France, il revêt cependant un caractère important pour le département des Yvelines dans lequel il est totalement inclus : il couvre $30 \%$ de sa superficie, et abrite $30 \%$ de sa population. Le bassin de la Mauldre forme un grand plateau, disséqué au niveau de la Mauldre moyenne et aval par des vallées peu profondes et peu encaissées. La qualité de l'eau est globalement meilleure en rive gauche amont qu'en rive droite ainsi que sur l'aval de la Mauldre (figure 1), selon les données disponibles dans le cadre de différents réseaux de mesure : le Réseau national de bassin (depuis 1983), le Réseau Tournant (1983 à 1990) et I'Inventaire national de la pollution (1971, 1976 et 1981). Ce bassin versant couvre des zones rurales agricoles et forestières, au sud et à l'ouest, et quelques petits et moyens centres urbains, massivement à l'est, en rive droite, dont les plus importants sont les agglomérations d'Élancourt, de Plaisir et de Versailles, situées à l'amont de petits affluents, les rus d'Élancourt, de Maldroit et de Gally. Le bassin de la Mauldre est très peuplé : on compte en 1990 un effectif total de population de 304110 habitants (INSEE, 1990), soit une densité moyenne communale de 934 hab $\mathrm{km}^{-2}$ avec, localement, des densités plus fortes qui atteignent entre 1500 et 2000 dans les sous-bassins de Gally et de Maldroit. Pour l'assainissement des eaux usées de ces collectivités, un parc de 23 stations d'épuration communales s'est peu à peu construit, avec une capacité théorique de traitement de 440900 Équivalents Habitants ${ }^{1}$ et une capacité réelle de 421375 (SATESE 78, 1995). Ces sta-

1. L'arrêté interministériel du 6 novembre 1996 établit une somme de ces paramètres à termes fixes pour estimer la production théorique de pollution par un habitant pour un jour, sur le bassin Seine-Normandie, à $162 \mathrm{~g}$. L'Équivalant Habitant (EH) ainsi défini servira à exprimer la charge de pollution qui entre dans une station d'épuration, qui y est traitée et qui en ressort, ji servira aussi pour déterminer le montant des taxes de pollution dues par les collectivités et les industriels aux Agences de l'eau. Les valeurs données pour les différents paramètres qui définissent l'Équivalent Habitant dans le cadre de l'arrêté du 06/11/96, fixé pour 5 ans à compter du 01 janvier 1997, sont les suivantes : $90 \mathrm{~g}$ de matières en suspension, $57 \mathrm{~g}$ de matières oxydables, 0,2 équitox de matières inhibitrices, $15 \mathrm{~g}$ d'azote réduit, $4 \mathrm{~g}$ de phosphore total, $0,05 \mathrm{~g}$ de composés organohalogénés absorbables sur charbon actif et 0,23 métox. 
tions abattent une partie importante de la pollution totale qui y est apportée (rendement global ${ }^{2}$ de $88 \%$ ), mais pour le phosphore et l'azote, les rendements sont faibles (respectivement $40 \%$ et $70 \%$ ), car une grande partie de ces stations sont anciennes et n'effectuent pas de traitements très poussés. La qualité des cours d'eau ${ }^{3}$ est donc dégradée par la pression anthropique qui s'exerce sur ce bassin versant (figure 1).

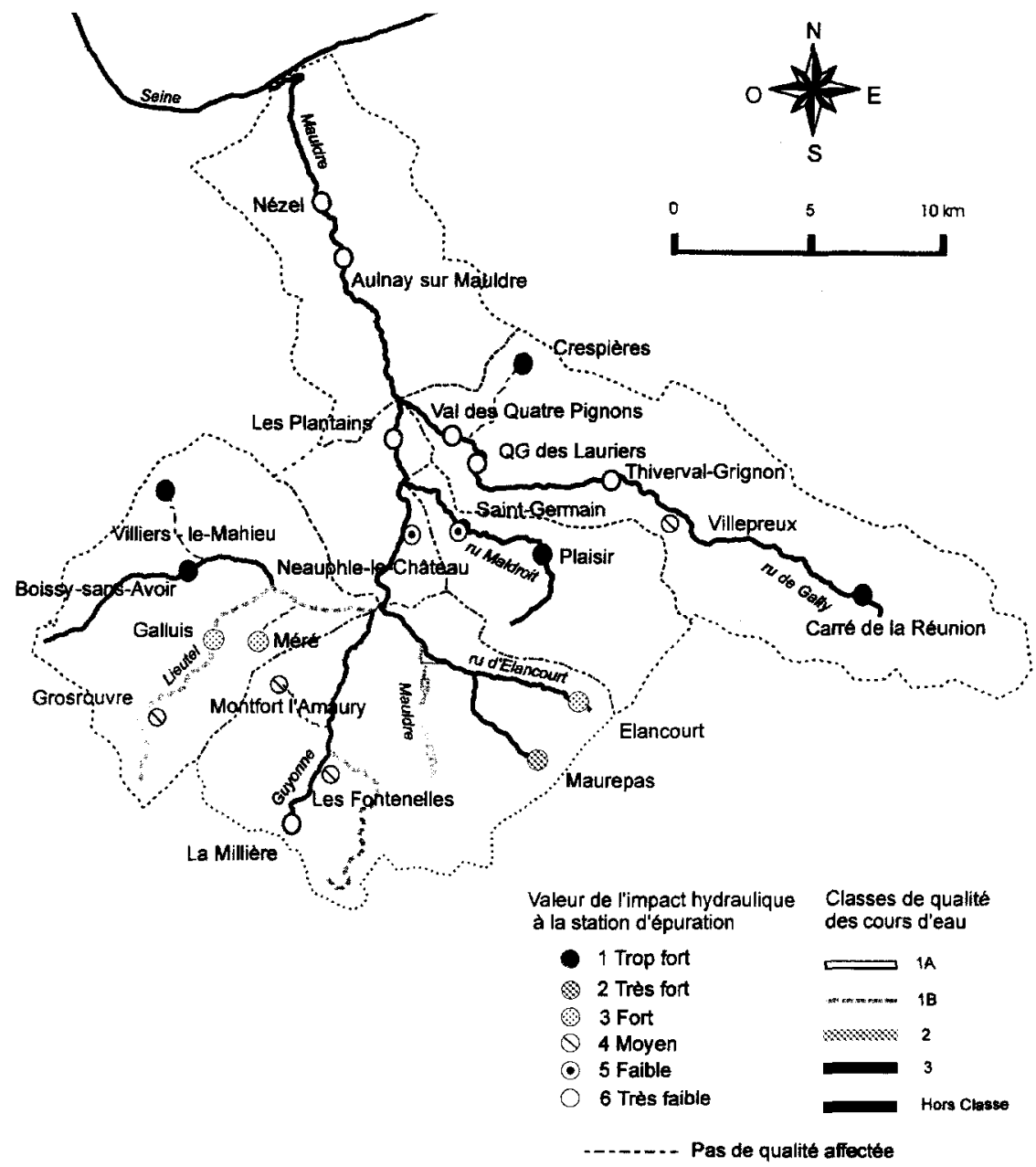

Figure 1 Qualité des cours d'eau et application de la méthode du ratio de dilution au bassin de la Mauldre (DIREN, 1994).

Mauldre river quality and dilution's ratio method application on the Mauldre river

2. Le rendement épuratoire d'une station correspond à la différence entre la pollution entrée et celle qui a été éliminée par les traitements, exprimée en \%.

3. La qualité des eaux de surface est appréhendée par les valeurs de différents paramètres regroupés en classes de valeurs représentatives soit de la qualité générale de l'eau, soit d'une aptitude à satisfaire un usage. Pour plus de détails, se reporter à l'étude bibliographique inter agences (AESN, 1991). 


\section{2 - MÉTHODOLOGIE}

Quatre méthodes ont été appliquées pour cette étude, avec pour chacune leurs intérêts et leurs limites.

Le ratio de dilution $(R)$, appelé aussi impact hydraulique, est couramment utilisé par les services du département chargés de l'environnement (DIREN Ilede-France, 1996). Pour chaque couple station d'épuration/rivière, il relie le débit caractéristique d'étiage du cours d'eau ( $Q$ d'étiage) au débit de rejet moyen annuel de la station (Q réel), calculé par le SATESE ${ }^{4}$. Le débit d'étiage est calculé par le service de la navigation s'il y a une station de jaugeage proche ; on parle alors de QMNA $5^{5}$. Sinon on utilise des données estimées, extraites du dossier d'objectif de qualité du cours d'eau. Le débit réel de la station d'épuration est calculé par le SATESE du département à l'aide de mesures moyennes sur 24 heures et de mesures ponctuelles. On obtient donc la formule suivante : $R=Q$ d'étiage/ $Q$ réel. On peut découper les valeurs $R$ en 6 classes (I) décrites dans le tableau 1 : si $R<1$, l'impact est très fort car la dilution des effluents est très faible, $1<=R<2$ l'impact est fort, $2<=R<5$ l'impact est moyen, $5<=R<10$ l'impact est faible et $R>=10$ l'impact est très faible car la dilution est ici très importante. L'inconvénient majeur de cette méthode est que l'on ne tient compte ni de la qualité de l'eau, ni du rejet, ni de la rivière: on a seulement une idée des possibilités de dilution de l'effluent dans le milieu récepteur (tableau 1).

Tableau 1 Classes de l'indice DIREN, avec les valeurs prises par le rapport de dilution, la valeur de l'impact hydraulique et son appréciation (DIREN, 1996).

Table 1 DIREN's index, values of the dilution's rate, value of the hydraulic impact and its description.

\begin{tabular}{|c|c|}
\hline $\begin{array}{c}\text { Rapport } \\
\text { de dilution (R) }\end{array}$ & $\begin{array}{c}\text { Valeur de l'Impact Hydraulique (I) } \\
\text { et appréciation }\end{array}$ \\
\hline$R<1$ & 1 - Trop fort \\
$1 \leq R<2$ & 2 - Très fort \\
$2 \leq R<5$ & 3 - Fort \\
$5 \leq R<10$ & 4 - Moyen \\
$10 \leq R<20$ & 5 - Faible \\
$R \geq 20$ & 6 - Très faible \\
\hline
\end{tabular}

2. Le rendement épuratoire d'une station correspond à la différence entre la pollution entrée et celle qui a été éliminée par les traitements, exprimée en \%.

3. La qualité des eaux de surface est appréhendée par les valeurs de différents paramètres regroupés en classes de valeurs représentatives soit de la qualité générale de l'eau, soit d'une aptitude à satisfaire un usage. Pour plus de détails, se reporter à l'étude bibliographique inter agences (AESN, 1991).

4. Le Service d'Assistance Technique aux Exploitants des Stations d'Épurations est un service du département chargé d'apporter une aide technique aux gestionnaires des stations lors de visites des ouvrages accompagnées de conseils, de prélèvements et d'un rapport de visite.

5. Il s'agit du débit minimal de 30 jours consécutifs pour une période de retour de 5 ans. 
L'indice TRABUC (T) ou «taux de pollution » est issu de l'Agence de l'eau Seine-Normandie (MORIZE, 1984). II relie le flux de pollution qui sort de la station d'épuration pour le ou les paramètres choisis au débit d'étiage de la rivière par la formule suivante : $T$ = Flux polluant rejeté par la station/Q d'étiage ( $T$ est exprimé en $\mathrm{mg} \cdot \mathrm{L}^{-1}$ ). Cet indice indique la contribution de la station étudiée à la qualité de la rivière, en terme de concentration pour chaque paramètre étudié, après dilution, c'est-à-dire en aval du rejet de la station. On estimera que le rejet de la station dégrade le cours d'eau si $T$ dépasse les valeurs admises pour la classe de qualité 1B (bonne qualité de l'eau). Cette méthode tient compte seulement de la qualité du rejet de la station, principalement parce que ce sont des données disponibles. En revanche, on ne tient pas compte de la qualité réelle du cours d'eau à l'amont du rejet et on présuppose qu'elle est bonne. Cet indice nous renseigne donc sur la dilution des polluants contenus dans le rejet ainsi que sur les concentrations apportées en rivière par la station.

Ces deux méthodes, le taux de dilution (R) et le taux de pollution (T), analysent de façon ponctuelle les relations entre le rejet d'une station et le cours d'eau récepteur. Les méthodes suivantes posent le problème à des échelles de plus en plus globales.

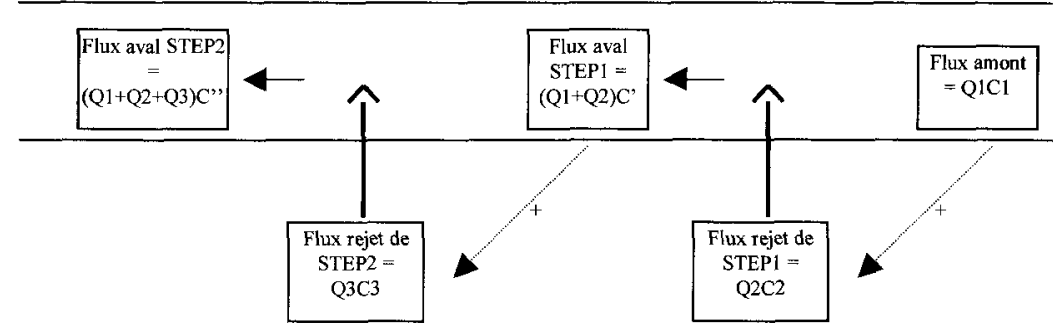

Figure 2 Bilan des flux en rivière en prenant en compte les flux de rejets des stations, par paramètre chimique étudié.

Pollutant load assessment in rivers, taking account waste water treatment plants outfall, for each studied chemical element.

avec:

Q1 = débit du cours d'eau en amont de la première station d'épuration

$\mathrm{C} 1$ = concentration du paramètre considéré en amont de la première station d'épuration

STEP1 = station d'épuration $n^{\circ} 1$

Q2 = débit en sortie de la première station d'épuration

$\mathrm{C} 2$ = concentration du paramètre considéré en sortie de la première station d'épuration

$\mathrm{C}^{\prime}=$ concentration du cours d'eau en aval du premier rejet de station d'épuration

STEP2 = station d'épuration $\mathrm{n}^{\circ} 2$

Q3 = débit en sortie de la deuxième station d'épuration

$\mathrm{C} 3=$ concentration du paramètre considéré en sortie de la deuxième station d'épuration

$C^{\prime \prime}=$ concentration du cours d'eau en aval du deuxième rejet de station d'épuration

On pose donc l'équation suivante à l'aval du premier rejet de station d'épuration pour connaître

le flux dans le cours d'eau:

$\mathrm{C}^{\prime}(\mathrm{Q} 1+\mathrm{Q} 2)=\mathrm{Q} 1 \mathrm{C} 1+\mathrm{Q} 2 \mathrm{C} 2$

$\Leftrightarrow C^{\prime} 1-C 1=(Q 1 C 1 / Q 1+Q 2)+(Q 2 C 2 / Q 1+Q 2)-C 1(Q 1+Q 2) /(Q 1+Q 2)$

On pose : $\triangle \mathrm{C}=\mathrm{C}^{\prime} 1-\mathrm{C} 1$

$\Leftrightarrow \Delta \mathrm{C}=\mathrm{Q} 2(\mathrm{C} 2-\mathrm{C} 1) /(\mathrm{Q} 1+\mathrm{Q} 2)$ 
La méthode du bilan des flux que nous avons mise au point prend mieux en compte les rejets des stations et la qualité des cours d'eau récepteurs car nous considérons ici la rivière dans son continuum et les rejets dans leur succession, grâce à un bilan global des flux (figure 2). De cette manière, nous sommes en mesure d'analyser les évolutions des débits et de la qualité de la rivière au fil de l'eau par des calculs par itération. La question posée trouve ainsi une réponse appréhendée de façon plus complète et plus globale que les ratios exposés plus haut. C'est de cette manière que cette méthode vise à pallier le manque de données existant sur les cours d'eau, directement en amont et en aval des rejets de chaque station d'épuration. Des mesures de terrain complètent les valeurs calculées pour estimer l'impact des stations d'épuration sur les cours d'eau. La variation de la qualité du cours d'eau induite par le rejet de chaque station d'épuration est établie de la façon suivante. Pour la station amont, on définit un flux amont de pollution en rivière: Q1C1. On connaissait celui rejeté par la station : Q2C2. On calcule ensuite le flux aval en rivière en postulant qu'il n'est que la somme des deux flux précédents : Q1C1 + Q2C2 $=C^{\prime}(Q 1+Q 2)$. Puis on procède par itérations successives pour explorer l'aval du cours d'eau. On peut alors calculer pour l'aval de chaque station la concentration ajoutée en rivière par le rejet $(\Delta \mathrm{C})$. Elle équivaut à la moyenne des flux amont de la station considérée (CiQi), pondérée par la somme des débits amont (Qi). Cette méthode laisse de côté certaines variables couramment étudiées dans les modèles, comme par exemple la transformation des flux rejetés en rivière dès leur arrivée en rivière ou peu après, les apports en eaux autres que les rejets de ces stations qui peuvent provenir de déversoirs d'orages, de rejets sauvages, du ruissellement de parcelles cultivées, d'échanges avec les nappes phréatiques pendant les basses eaux surtout. C'est en cela que cette méthode de bilan des flux se différencie nettement d'un modèle complet à trois dimensions. II s'agit d'un modèle ponctuel, le plus simple. II permet d'évaluer la qualité du milieu à l'aval immédiat du point de rejet de la station d'épuration à un instant donné et en même temps la qualité moyenne du cours d'eau par itération sur des tronçons successifs, en faisant l'hypothèse d'un régime permanent. II constitue un modèle de base unidimensionnel qui décrit globalement le cours d'eau en un continuum (figure 2).

\subsection{Données et application de la méthode}

Les calculs ont été effectués sur le ru de Gally et ses cinq stations d'épuration, pour deux paramètres, le phosphore total (Ptotal) et l'ammonium $\left(\mathrm{N}_{-} \mathrm{NH}_{4}\right)$, qui traduisent bien le type de pollution présente sur le bassin de la Mauldre, la pollution domestique. Un autre intérêt du paramètre phosphore est qu'il est conservateur, c'est-à-dire qu'il ne subit que peu de transformations dans un cours d'eau de la taille du ru de Gally. Le débit de base du ru de Gally en amont de la station d'épuration du Carré de Réunion est estimé à $1 \mathrm{~L} \cdot \mathrm{s}^{-1}$ en étiage. Il est en effet très faible car il n'est alimenté que par la mare de la Ferme de Gally, elle-même alimentée en été par un puits situé dans la ferme ; en période hivernale les surverses des eaux du Grand Canal du Château de Versailles l'alimentent également. Nous avons estimé les concentrations des deux paramètres à la borne inférieure de la classe 3 de la grille de qualité générale de 1971 pour l'azote, et de la grille eutrophisation de $1990\left(2 \mathrm{mg} \cdot \mathrm{L}^{-1}\right.$ pour le $\mathrm{N}$ $\mathrm{NH}_{4}$ et $0,6 \mathrm{mg} \cdot \mathrm{L}^{-1}$ pour le Ptotal), car les eaux provenant de la ferme sont de mauvaise qualité, compte tenu qu'elles stagnent longtemps dans la mare. De 
plus, cette classe correspond à l'objectif de qualité fixé pour ce ru dans le dossier d'objectif de qualité du bassin de la Mauldre. L'exercice consiste donc à regarder si les rejets des stations d'épuration parvenant à ce ru, tels qu'ils sont mesurés par le SATESE 78, peuvent permettre de respecter l'objectif de qualité du ru (classe 3 ), tout au long de son cours. Les données concernant les stations d'épuration utilisées ici proviennent des moyennes des visites de l'année 1995 par le SATESE 78. Les données du milieu naturel proviennent du dossier d'objectif de qualité pour la définition de la classe d'objectif de 1975. Les mesures de débit d'étiage (QMNA5) utilisées pour l'amont de la station du Val des Quatre Pignons sont fournies par la DIREN Ile-de-France (Subdivision Réseau de Mesures et Hydrométrie - Banque HYDRO). Les flux dans le ru sont calculés au fil de l'eau. On postule dans un premier temps qu'il n'y a pas d'autres apports en eau dans le ru que le débit, qui vient de l'amont de la station. À ce débit s'ajoutent les débits successifs des rejets des stations (hypothèse 1 avec un débit amont de $1 \mathrm{~L} \cdot \mathrm{s}^{-1}$ et hypothèse 2 avec $10 \mathrm{~L} \cdot \mathrm{S}^{-1}$ ). Ne connaissant pas les apports latéraux qui aboutissent au ru de Gally, on ajuste dans un deuxième temps le débit aval du ru à l'aide des données fournies par la station de jaugeage du Val des Quatre Pignons (hypothèse 3).

L'indice de stress démographique, de l'anglais «population stress index " (MEYBECK, 1998a), est le ratio de la population vivant sur le bassin considéré divisé par le débit du cours d'eau à son exutoire, exprimé en hab. $\mathrm{L}^{-1} \cdot \mathrm{s}$. Des valeurs de référence ont été établies en fonction du type d'occupation du sol du bassin versant (naturel, agricole, rural, urbain). Cette méthode prend en compte la relation rejet de station/cours d'eau à un niveau amont.

\section{3 - RÉSULTATS ET INTERPRÉTATIONS}

Les différentes méthodes ont été appliquées au bassin versant de la Mauldre, avec les outils disponibles, pour évaluer la pression anthropique qui s'exerce sur ces cours d'eau:

- à l'aval des stations d'épuration, il s'agit d'impacts analysés de manière ponctuelle sur les cours d'eau par les deux premières méthodes ;

- au fil de la rivière, ces impacts seront analysés par la méthode du bilan des flux puis par celle du bilan global des impacts ;

- et à l'aval de chaque affluent de la Mauldre, cette pression est évaluée à l'aide de l'indice de stress démographique.

\subsection{Impacts ponctuels}

Les impacts ponctuels sont estimés à partir de deux méthodes : le ratio de dilution et le taux de pollution.

\subsubsection{Ratio de dilution}

Une valeur d'impact hydraulique pour chaque station d'épuration du bassin a été calculée, à l'aide des données des débits réels de ces stations et des débits d'étiage des cours d'eau; une représentation spatiale en est faite sur la 
figure 1. On obtient un premier ordre de grandeur de l'impact du rejet de ces stations pendant les périodes critiques pour la vie aquatique. Globalement, les cours d'eau à faibles débits d'étiage sont les plus menacés par les stations d'épuration. En effet, les débits de rejets des stations sont souvent plus importants que les débits des milieux récepteurs : il s'agit en particulier des rus de Crespières, du Breuil, de Maldroit, d'Heudelimay qui affichent tous un impact de 1. Les débits des tronçons aval de la Mauldre sont moins influencés par ceux qui sont rejetés par les stations d'épuration. Ainsi avec un impact de 6 pour toute la partie aval de la Mauldre, les dilutions des rejets de stations peuvent être considérés comme moins problématiques puisque l'eau de la rivière est de mauvaise qualité et que les rejets de station sont peu importants.

\subsubsection{Cas du ru de Gally (tableau 2)}

Son débit amont est essentiellement constitué par celui du rejet de la station d'épuration du Carré de Réunion. Pour des commodités de calculs, nous avons considéré ici que le débit d'étiage du ru, en amont de la station, était non nul mais très faible, égal à $100 \mathrm{~m}^{3} \cdot \mathrm{j}^{-1}$. L'impact de cette station sur le ru est donc sans commune mesure avec les autres stations, mais sa valeur $(l=1)$ ne permet pas de différencier ce cas des autres. Pour s'en apercevoir, il faut regarder la valeur prise par le rapport entre les débits (qui a servi à calculer l'indice) qui est très proche de zéro $(R=0,002)$. L'impact hydraulique des stations plus en aval s'amenuise ( $I=$ moyen à très faible) et le rapport de dilution croît ( $R=7,3$ à 501) jusqu'à la confluence du ru avec la Mauldre. Le gros du débit de ce ru est ainsi rapidement constitué et les apports des stations suivantes sont peu significatifs.

Tableau 2 Application de la méthode du ratio de dilution au ru de Gally. Sources : DIREN, SATESE.

Table 2 The dilution's ratio method application to the river Gally.

\begin{tabular}{|c|c|c|c|c|c|}
\hline $\begin{array}{l}\text { Nom de la station } \\
\text { d'épuration }\end{array}$ & $\begin{array}{l}\text { Q théorique } \\
\text { STEP }\left(\mathrm{m}^{3} \cdot \mathrm{j}^{-1}\right)\end{array}$ & $\begin{array}{c}\text { Q réel } \\
\operatorname{STEP}\left(m^{3} \cdot j^{-1}\right)\end{array}$ & $\begin{array}{c}\text { Q étiage } \\
\text { cours d'eau } \\
\left(\mathrm{m}^{3} \cdot \mathrm{j}^{-1}\right)\end{array}$ & $\begin{array}{l}\mathbf{R}=\text { Valeur } \\
\text { du rapport }\end{array}$ & $\begin{array}{l}\text { I = Valeur et } \\
\text { appréciation } \\
\text { de l'Impact } 0 \\
\text { d'étiage }\end{array}$ \\
\hline Carré de Réunion & 53500 & 39467 & 100 & 0,002 & 1 - Trop fort \\
\hline Villepreux & 6500 & 5856 & 42768 & 7,3 & 4-Moyen \\
\hline Thiverval-Grignon & 1800 & 705 & 49507 & 70 & 6 -Très faible \\
\hline $\begin{array}{l}\text { Val des Quatre } \\
\text { Pignons }\end{array}$ & 555 & 316 & 56160 & 178 & 6 - Très faible \\
\hline Q.G. des Lauriers & 225 & 130 & 56160 & 501 & 6 - Très faible \\
\hline
\end{tabular}

Si les valeurs de l'impact hydraulique vont en augmentant de l'amont vers l'aval, cela veut dire que l'impact des stations sur le ru s'amenuise seulement du point de vue quantitatif. Et c'est sans prendre en compte les paramètres de la qualité de l'eau, ce que nous examinerons ci-dessous (tableau 2). 


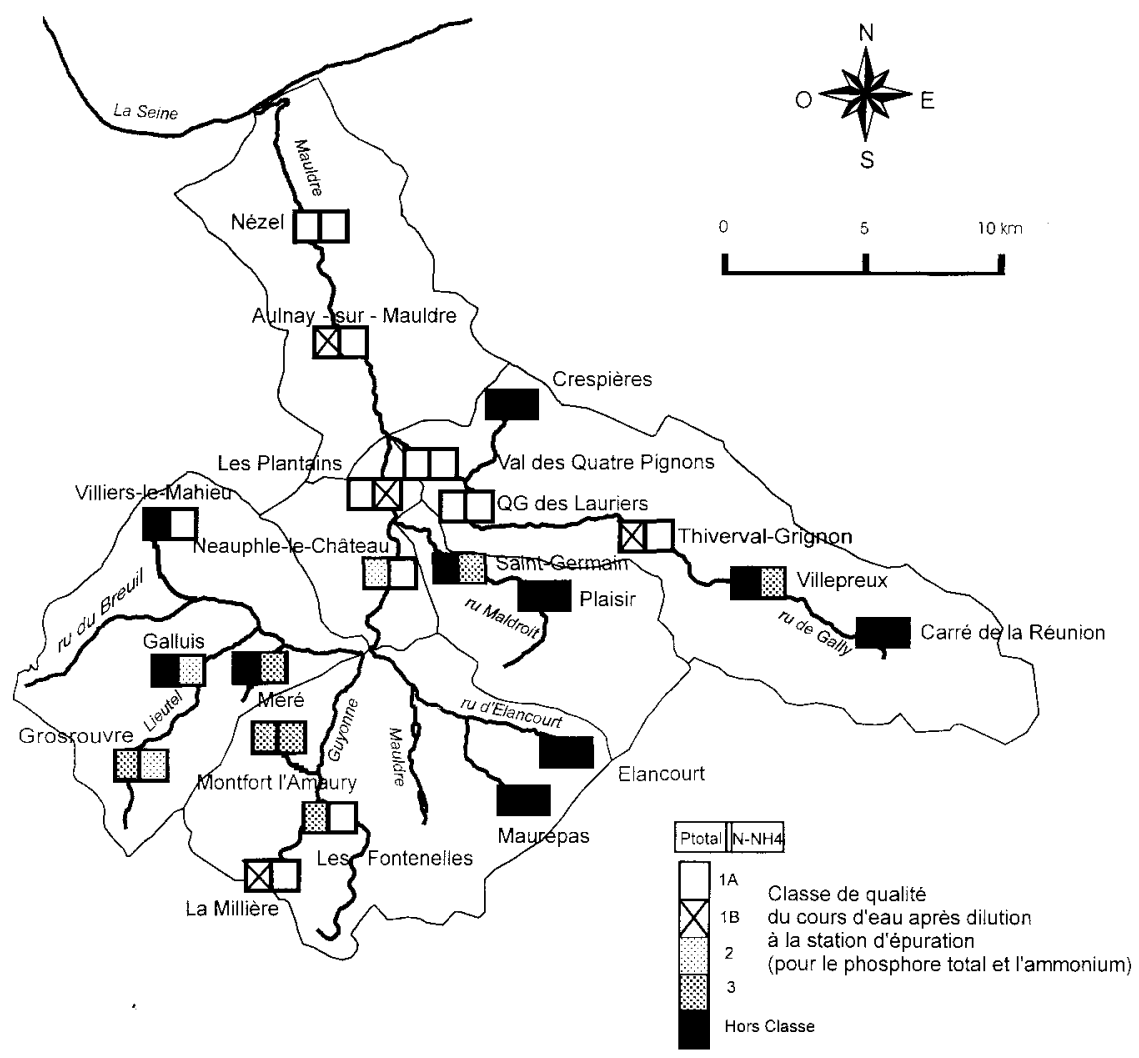

Figure 3 Indice Trabuc pour les stations d'épuration de la Mauldre.

Pollution's rate method application on the river Mauldre.

\subsubsection{Taux de pollution (Indice TRABUC)}

Cet indice est témoin de l'impact des rejets des stations sur la qualité des eaux de surface. Appliqué aux données de la Mauldre (figure 3), il montre une très forte influence des rejets sur l'amont des cours d'eau aussi bien en azote qu'en phosphore. Cet impact s'amenuise sur le cours aval de la Mauldre et il est même nul à la station la plus en aval, Nézel, ainsi que pour les trois stations de l'aval du ru de Gally. Les deux ratios de dilution présentent la même tendance : les impacts les plus forts sont en amont et s'amenuisent en aval. Grâce à l'application de cette méthode, on peut conclure que la qualité des tronçons de cours d'eau s'améliore en aval du ru puisqu'elle est moins influencée par les rejets polluants des stations d'épuration. C'est un résultat, hélas, en contradiction avec les mesures de terrain que nous avons effectuées par ailleurs, il ne peut donc nous satisfaire. En effet, l'indice TRABUC tient mieux compte de la qualité de l'eau que l'indice DIREN. Cependant il n'existe aucune donnée sur la qualité du cours d'eau récepteur. L'indice TRABUC, lui, postule qu'il s'agit d'une bonne qualité. Or cette bonne qualité n'existe pas sur l'aval de la Mauldre, ni même en amont de certains de ses affluents. L'application de la 
méthode suivante permet de décrire l'évolution de la qualité de cette eau en intégrant les données existantes sur les rejets successifs des stations.

\subsection{Bilan des flux (test sur le ru de Gally)}

Les calculs effectués par cette méthode montrent une évolution de la qualité du ru très fortement marquée par le rejet de la station d'épuration du Carré de Réunion (figure 4). Si l'on considère l'hypothèse 1, les concentrations en ammonium dans le ru doublent après ce premier rejet, passant de $2 \mathrm{mg} \cdot \mathrm{L}^{-1}$ en amont à $4 \mathrm{mg} \cdot \mathrm{L}^{-1}$ en aval, et les concentrations en phosphore total augmentent beaucoup, passant de $0,6 \mathrm{mg} \cdot \mathrm{L}^{-1}$ en amont de la station à $10 \mathrm{mg} \cdot \mathrm{L}^{-1}$ en aval : on est Hors Classe selon la grille de qualité. Jusqu'à l'aval, elles ne varient que peu significativement autour de $9,7 \mathrm{mg} \cdot \mathrm{L}^{-1}$. Les concentrations en ammonium doublent encore une fois à la sortie de la station de Villepreux, au lieu dit la Ferme de Mézu, pour atteindre $8 \mathrm{mg} \cdot \mathrm{L}^{-1}$, si bien que le ru de Gally est " déclassé », et sa qualité de classe 3 issue du dossier d'objectif de qualité passe alors Hors Classe, selon la grille de qualité de 1971. Puis les concentrations augmentent encore très légèrement mais peu significativement : l'imprécision des différentes données utilisées et du modèle simple de calcul n'autorise pas à prendre en compte des variations de concentration du $10^{\mathrm{e}} \mathrm{de}$ $\mathrm{mg} \cdot \mathrm{L}^{-1}$. Le déclassement du ru s'effectue pour les deux paramètres étudiés. L'objectif de qualité est donc très loin de pouvoir être respecté à cause des rejets des stations en étiage. Les deux autres hypothèses nous donnent des résultats semblables. II est possible d'étendre ces calculs aux paramètres suivants : Matières En Suspension, Demande Biologique en Oxygène pour 5 jours, Demande Chimique en Oxygène et azote kjeldhal, pour lesquels il existe des données de concentration mesurées dans les rejets des stations d'épuration, des préconisations pour les normes de rejet (différents niveaux) et des indications d'objectifs de qualité. Seul l'azote kjeldhal semble être un paramètre intéressant à ajouter à cette étude car les autres subissent trop de variations dans la rivière, au fil de l'eau et du temps. La pollution physicochimique n'est qu'un aspect de la dégradation de la qualité des eaux superficielles. La contamination par métaux lourds et bactéries pathogènes en est un autre qu'il serait par exemple intéressant d'étudier car ces polluants ont souvent pour origine des rejets de stations d'épuration. Ils peuvent s'avérer toxiques pour la faune, la flore et l'homme.

Nous venons de voir que les concentrations et les flux en rivière calculés pour les paramètres de pollution domestique augmentent au fil de l'eau. On observe ce même phénomène avec les mesures effectuées dans le ru durant une campagne 24 heures le 17 septembre 1998 (figure 4). La méthode du bilan des flux peut donc être validée. La qualité de l'eau se dégrade dès l'amont avec le rejet de la station de Carré de Réunion. Les autres rejets ne participent que très peu à la pollution du ru. Les ratios de dilution ne peuvent donc pas être utilisés pour estimer la pression anthropique sur un cours d'eau à l'échelle d'un bassin versant. Si des mesures en rivière font défaut, la méthode de calcul de flux donne un résultat plus proche du fonctionnement de la rivière, observé avec nos mesures 24 heures. Elles intègrent également différents rejets de stations d'épuration. Compte tenu de l'ensemble de ces éléments, il nous a paru particulièrement utile d'estimer l'impact global des rejets de stations d'épuration sur le milieu étudié. À cet effet une analyse systémique a été conduite. 


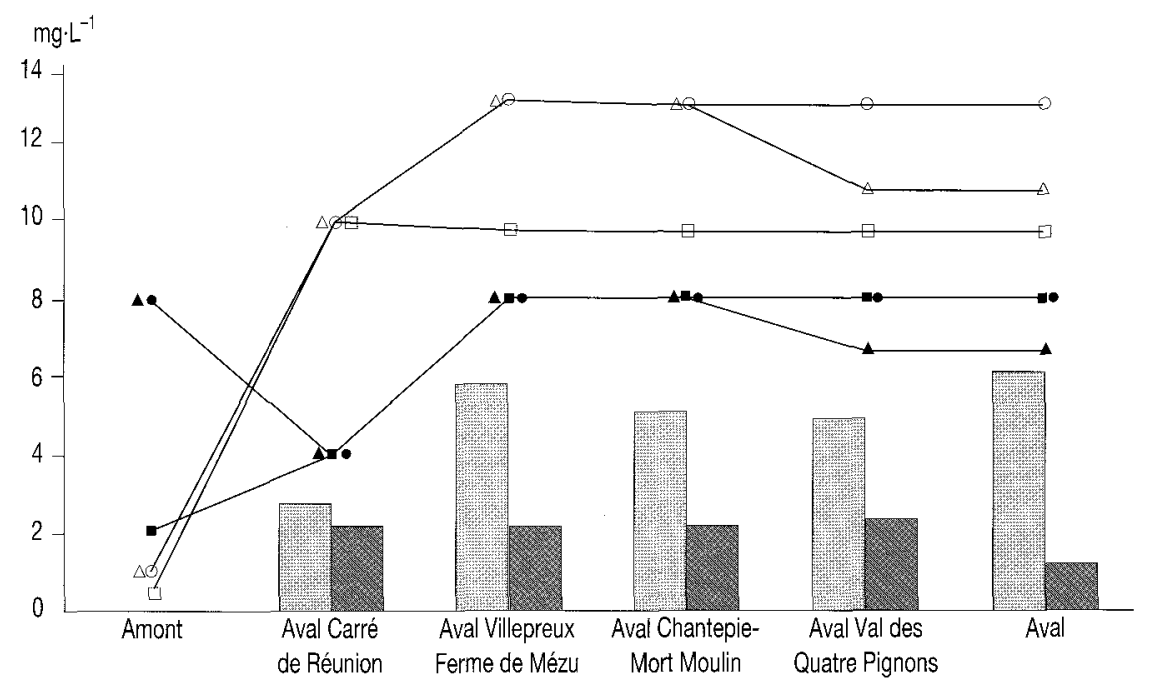

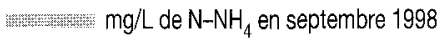

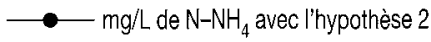

$\mathrm{mg} / \mathrm{L}$ de $\mathrm{P}-\mathrm{PO}_{4}$ en septembre 1998

$-\_-m g / L$ de $P$ total avec l'hypothèse 2

_.__ $\mathrm{mg} / \mathrm{L}$ de $\mathrm{N}-\mathrm{NH}_{4}$ avec l'hypothèse 1

$\longrightarrow \mathrm{mg} / \mathrm{L}$ de $\mathrm{N}-\mathrm{NH}_{4}$ avec l'hypothèse 3

$\square \mathrm{mg} / \mathrm{L}$ de $P$ total avec l'hypothèse 1

$\triangle \mathrm{mg} / \mathrm{L}$ de $\mathrm{P}$ total avec l'hypothèse 3

Hypothèse 1 avec $Q=1 \mathrm{~L} / \mathrm{s}$

Hypothèse 2 avec $Q=10 \mathrm{~L} / \mathrm{s}$

Hypothèse 3 avec $Q$ ajusté à la station de jaugeage du Val des Quatre Pignons

Figure 4 Concentrations dans le ru de Gally en phosphore total et en ammonium, mesurées (moyennes 24 heures du 17 septembre 1998) et calculées selon les 3 hypothèses de la méthode du bilan des flux.

Sources : DIREN, Laboratoire de Géologie appliquée (Paris VI).

Total phosphorus and ammonium concentrations in the river Gally, measured (mean of 24 hours measurement the 17 September 1998) and calculated with the pollutant rate method's tree hypothesis.

\subsection{Bilan global des impacts}

\subsubsection{L'approche " systèmes "}

Pour transcrire les descriptions qui précèdent en système et construire un bilan global, il est nécessaire d'agrandir le périmètre autour de la question des relations entre les rejets des stations d'épuration et les cours d'eau récepteurs. Cette approche systémique a deux principaux effets. D'une part elle permet d'obtenir de nombreux autres points de vue sur l'objet d'étude, d'autre part on voit également mieux apparaître les relations entre les éléments discutés plus haut et il est possible d'en faire apparaître d'autres.

Dans un premier temps apparaissent des relations à l'intérieur du système "stations d'épuration ", puis entre ce système et d'autres systèmes qui sont pertinents à l'échelle du bassin versant. Sur la base du modèle de représenta- 


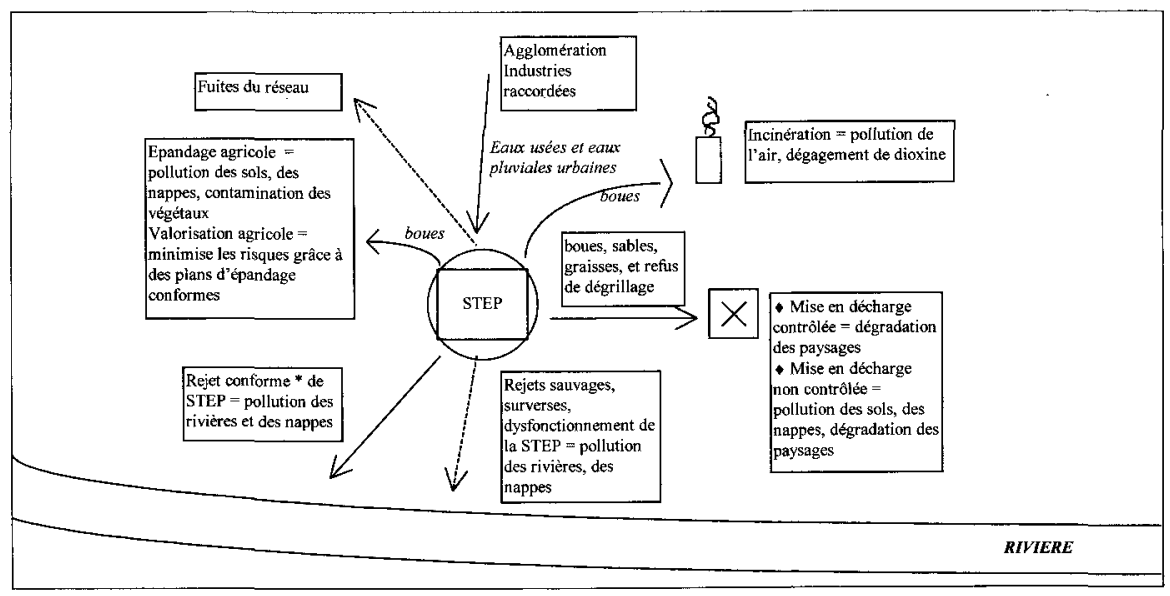

Figure 5 Schéma simplifié des relations qui se nouent entre la station d'épuration, la rivière et le bassin versant.

Basic diagram of the relations between waste water treatment plants, river and catchment.

tion du monde de ROSNAY (1975), une approche systémique de l'objet étudié est proposée. On distingue successivement les aspects structural et fonctionnel du système (figure 5). En d'autres termes chaque système est abordé par ce qu'il est, ce qu'il fait et ce qu'il devient. Les éléments du système, ses frontières et ses liaisons internes sont transcrites.

En appliquant cette méthode systémique à l'analyse d'une station d'épuration (tableau 3), on voit surgir une quantité importante de relations avec d'autres éléments que le cours d'eau récepteur. L'espace géographique à prendre en compte s'élargit jusqu'aux limites du bassin versant et de l'environnement atmosphérique (frontières du système), les éléments pris en compte dans le système nous font remonter à l'origine de la production du flux amené dans la station d'épuration, c'est-à-dire à la population et aux industries qui sont raccordées au réseau communal d'assainissement. Les aspects suivants du système montrent combien ces éléments sont importants.

L'aspect structural du système, c'est-à-dire l'organisation dans l'espace de ses éléments, comporte essentiellement les frontières, les éléments, les réservoirs et les réseaux de communication.

Les frontières qui séparent le système étudié du monde extérieur sont les limites du bassin de collecte de la station d'épuration et du milieu naturel qui reçoit les effluents épurés (cours d'eau superficiel puis parfois nappe souterraine). Les éléments (ou composants) peuvent être dénombrés et assemblés en catégories. II s'agit ici de la station d'épuration, de tous ses composants (bassins, canalisations, silos, éléments chimiques, particules, biomasses), des résidus de l'épuration, des points de rejet des effluents épurés ou non, des habitants et industriels raccordés aux stations qui produisent ces effluents, des composantes du climat. II s'agit aussi des acteurs du système. Les réservoirs rassemblent les éléments dans lesquels sont stockés de l'énergie, de l'informa- 
Tableau 3 Application du Macroscope à l'étude d'un bassin versant et des rejets de ses stations d'épuration.

Table 3 The Macroscope's application on the Mauldre catchment and its waste water treatment plants.

Aspect structural

Frontières

Éléments

Réservoirs

Réseaux

de communication

\section{Aspect fonctionnel}

Flux

Vannes

Délais

Rétroactions positives et négatives
Contours des bassins

Environnement atmosphérique limité

Stations d'épuration. Éléments physiques et chimiques contenus dans les eaux usées dans le réseau et la station.

Climat

Points des rejets en rivière des stations

Population et industries raccordées - équivalents habitants Acteurs de la gestion.

Stations. Atmosphère

Bassins de stockage des eaux usées et pluviales

Canalisations. Déversoirs d'orage et trop-pleins. Cours d'eau Réseaux informatiques

Flux d'énergie physique qui permet la circulation des fluides et assure les échanges d'énergie et de matière Flux de matière. Flux de pollution. Flux de population Flux de données, d'informations et flux financiers

Débitmètres et déversoirs. Prélèvements et collectes Améliorations technologiques et épuration (ex. : la microfiltration) Normes et législations environnementales. Décisions. Aménagements

Temps de passage de l'eau et des polluants dans la STEP Temps de mélange des eaux de la STEP avec celles du cours d'eau

Temps de mise en place (réglementations et aménagements nouveaux, améliorations techniques)

Débordement de réseau (orage exceptionnel sur réseau unitaire) Processus d'autoépuration

Évolution de la population raccordée et raccordable visible dans l'évolution du POS qui détermine le dimensionnement de la station

Évolution de la législation pour les normes de rejets des effluents et des boues

Evolution des process industriels et de leurs rejets

Apparition/Disparition de rejets d'industries

Rénovation de réseau, de station et divers aménagements

Cycles nycthéméraux, saisonniers, climatiques

tion, des matériaux. On compte dans le système étudié la station, ses éléments et l'atmosphère. Les réseaux de communication permettent les échanges d'énergie, de matière et d'information entre les éléments et les réservoirs du système. Ces réseaux prennent ici la forme de canalisations qui véhiculent les effluents depuis le lieu de production jusqu'à celui de leur rejet dans le milieu naturel. II s'agit aussi du réseau de communication de l'information entre les différents acteurs. 
L'aspect fonctionnel du système distingue les flux, les vannes, les délais et les rétroactions. Les flux concernent l'énergie, l'information et les éléments qui circulent entre les réservoirs. Ils s'expriment comme les débits. Dans notre système, on parlera de flux atmosphériques, de flux polluants d'eau et de résidus de l'épuration, des flux de population mais aussi des flux de données et d'information sur les éléments du système ainsi que des flux financiers. Les vannes contrôlent les débits des différents flux. Chacune peut être visualisée comme un centre de décision qui reçoit des informations et les transforme en actions. On trouve dans le système étudié les débitmètres en entrée et en sortie de station, les différents déversoirs, mais aussi les améliorations techniques, les évolutions de la législation, les décisions d'aménagements, les diverses décisions de financement. Les délais résultent des vitesses différentes de circulation des flux, des durées de stockage dans les réservoirs. Ils jouent un rôle majeur dans les phénomènes d'amplification ou d'inhibition, typiques du comportement d'un système complexe. II s'agit ici du temps d'arrivée de l'eau usée dans le réseau, de son acheminement vers la station puis de son traitement (ce qui influence la transformation des paramètres polluants), du temps nécessaire au mélange de l'eau rejetée avec l'eau de la rivière ou encore nécessaire au mélange des boues avec le sol dans le cas de l'épandage. Pour ce qui est de l'eau de pluie qui entre dans un réseau unitaire, on a un temps de réponse des différentes surfaces avant que l'eau n'arrive dans le réseau. Le temps de mise en place des réglementations, des nouvelles techniques et des travaux d'aménagement est aussi à prendre en compte. Par exemple un retard d'application d'une nouvelle technique de traitement qui coïncide avec une augmentation du flux de population va pouvoir amplifier l'impact du rejet de la station sur le cours d'eau.

Les boucles de rétroaction jouent un rôle déterminant dans le comportement d'un système en combinant les effets de réservoirs, de délais, de vannes et de flux. En voici deux exemples. Si l'on a un réservoir de station unitaire et qu'un gros orage survienne, les temps de réponse des surfaces de ruissellement du réseau sont rapides et le réseau peut déborder. C'est une boucle de rétroaction positive. Ce type de boucle repose sur toute la dynamique du changement d'un système : croissance de la population raccordée et raccordable, évolution de la législation, des process industriels, apparition/disparition des rejets polluants, rénovation de réseaux ou de stations, aménagements divers. Second exemple, si l'effluent en entrée de station est accidentellement très pollué et non conforme à ce que la station traite habituellement, une partie de la biomasse des boues activées meurt et le rendement de la station baisse jusqu'au moment où les biocénoses se reforment. C'est une boucle de rétroaction négative. Ce type de boucle repose sur la régulation et la stabilité des équilibres, et notamment dans ce système sur les processus de l'autoépuration, poussée à l'extrême en station ou naturelle en rivière.

De cette approche systémique, il ressort que la pression exercée par les stations d'épuration est en fait un résidu de la pression initiale qui pèse sur le bassin versant, c'est-à-dire de la démographie ainsi que de certaines activités associées comme les industries. C'est le facteur démographie qui est dans ce cas le plus important. L'indice de stress démographique relie des données sur le milieu et des données démographiques. C'est pour ajouter cette dimension à notre étude qu'il va donc être utilisé. 


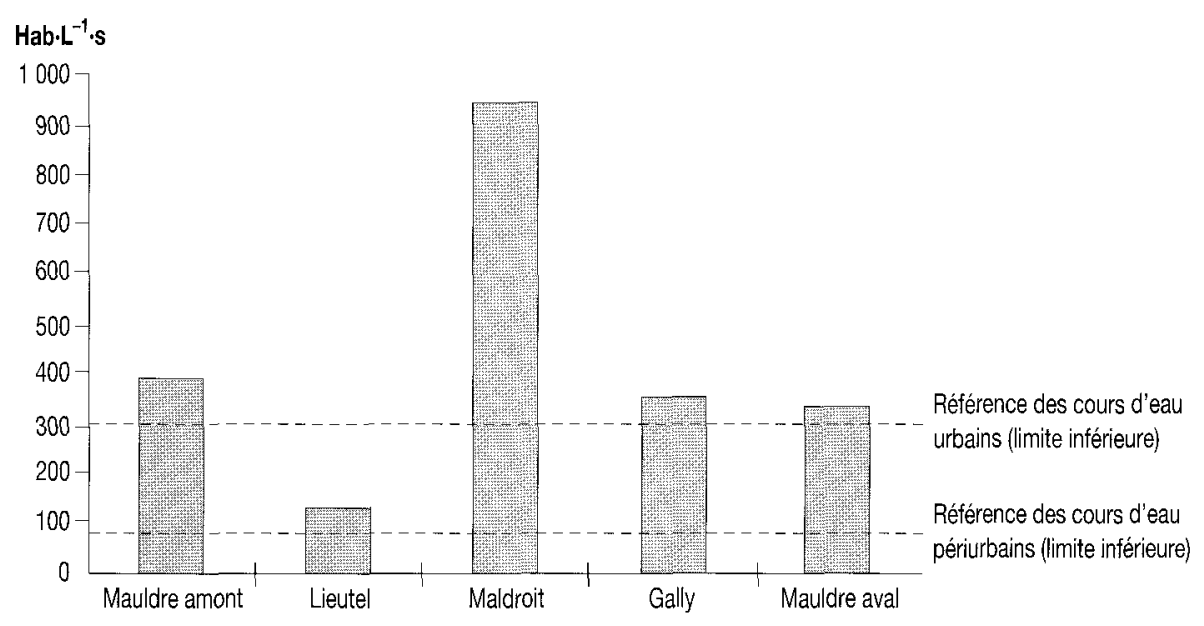

Figure 6 Indices de stress démographique, de la Mauldre et de ses principaux affluents, calculés pour la période d'étiage, avec référence à la typologie établie par MEYBECK, 1998a.

Sources: DIREN, INSEE, MEYBECK, 1998a.

Population stress index for each tributaries of the river Mauldre, calculated for a low flow period, with references to the MEYBECK's typology.

\subsubsection{Indice de stress démographique}

Les analyses effectuées sur cinq sous-bassins de la Mauldre (figure 6) nous montrent un indice extrêmement fort pour le ru de Maldroit (supérieur à 900 hab $L^{-1} . s$ ) et très fort pour le ru de Gally, l'amont de la Mauldre et la Mauldre aval (entre $300 \mathrm{hab} \cdot \mathrm{L}^{-1} \cdot \mathrm{s}$ et $400 \mathrm{hab} \cdot \mathrm{L}^{-1} \cdot \mathrm{s}$ ). Le premier sous-bassin se rapproche du type des cours d'eau urbains définis par MEYBECK (1998a), les trois suivants sont en limite de ce type et de celui des cours d'eau périurbains. La valeur de cet indice est bien plus faible sur le Lieutel (proche de $100 \mathrm{hab} \cdot \mathrm{L}^{-1} \cdot \mathrm{s}$ ), ce qui le place nettement dans le type des cours d'eau périurbains. Cet indice nous donne une idée globale de l'impact potentiel des populations vivant sur chaque sous-bassin de la Mauldre. Mais il postule que la pollution qui arrive à la rivière en provenance des activités humaines est proportionnelle à la densité de population qui vit sur ce bassin. Cette hypothèse se vérifie lorsque l'on s'attache seulement à donner des ordres de grandeur de la pression qui s'exerce sur un cours d'eau. Elle donne des résultats moins fiables si une pollution majeure provient des rejets d'une industrie en rivière par exemple, ce que cet indice ne peut prendre en compte.

\section{4 - CONCLUSION ET PERSPECTIVES}

Nous venons de passer en revue quelques méthodes pour appréhender les relations entre les rejets des stations d'épuration et les cours d'eau pour amé- 
liorer la compréhension d'un milieu sous très haute pression de pollution comme la Mauldre : ratio de dilution, taux de pollution, bilan des flux chimiques, systémique et indice de stress démographique.

L'application du ratio de dilution et du taux de pollution ne donne pas de résultats satisfaisants car ces méthodes nous fournissent des résultats ponctuels (à chaque station); ils occultent et nient le continuum de la rivière. Ces deux méthodes produisent des résultats de même tendance : les impacts les plus forts se situent en amont des cours d'eau et s'amenuisent à l'aval (I = 1 à l'amont du ru de Gally et 6 à l'aval). Ces résultats se sont avérés contraires à nos observations sur le terrain lors d'une campagne de mesures sur 24 heures. Mais il faut se garder de conclure hâtivement que la qualité de ces cours d'eau s'améliore de l'amont à l'aval, comme le montre la méthode du ratio de pollution. Des mesures en rivière nous ont montré que la qualité de l'eau se dégrade à l'aval. On a donc atteint les limites de ces deux premières méthodes qui ne prennent pas en compte la qualité du cours d'eau, l'application de la méthode du ratio de pollution conduit même à postuler une bonne qualité. La méthode du bilan des flux est plus intéressante car elle constitue un premier pas vers une approche globale dont l'intérêt se comprend mieux avec l'application de la méthode du Macroscope. On prend ici en compte la qualité amont de la rivière mais la validation de cette méthode par les mesures de terrain n'est pas satisfaisante (figure 4). On retrace bien l'évolution de cette qualité au fil de l'eau avec une augmentation de la pollution vers l'aval mais le calcul n'intègre pas assez de paramètres pour être bien représentatif des phénomènes qui se produisent en rivière.

L'approche systémique de la question posée par cet article nous permet de mieux appréhender la compréhension des phénomènes en jeu sur le bassin versant. Elle consiste à intégrer dans l'analyse un grand nombre d'éléments et à les mettre en relation. Le périmètre à prendre en compte est très vaste. Cette méthode demande l'acquisition de peu de données en comparaison des autres ; elle replace le problème posé dans un autre contexte et permet d'y apporter une réponse intéressante. Une relation intéressante se dégage entre la population d'un bassin et le débit d'étiage du cours d'eau avec l'indice de stress démographique. La relation entre les rejets des stations et les cours d'eau récepteurs mériterait donc d'être appréhendée avec un périmètre plus large. Remonter à l'échelle du bassin versant du cours d'eau ou de la Zone de Collecte d'Épuration étudiée permet de dégager une relation entre la densité démographique et le débit des cours d'eau du même bassin. Cette relation peut être considérée comme un bon indicateur de la pression anthropique qui s'exerce sur les milieux. Les modèles existants ou en projet sur les petits cours d'eau pour appréhender cette relation restent intéressants dans la mesure où ils étudient les transformations physicochimiques et/ou biologiques de l'amont à l'aval des cours d'eau (WOLFF, 1994). Mais ils pourraient avantageusement être complétés par des analyses de type systémique.

Le problème majeur que pose l'analyse des relations entre les rejets des stations d'épuration et la qualité des cours d'eau récepteurs de petite taille est le manque de données sur le milieu naturel et sur les stations en quantité suffisantes pour faire des analyses pertinentes : des données ponctuelles ou des moyennes annuelles s'avèrent induire un biais dans les conclusions. Devant ce manque de données on peut proposer d'orienter les recherches sur ce sujet vers un indicateur intégrateur de l'importance et de la variabilité de la pollution 
en station et en rivière. On peut penser à un indicateur biologique type Indice de Qualité Biologique Globale Normalisé (IQBGN) ou un indice diatomique.

Les indices biotiques sur invertébrés tels que I'IQBGN sont les plus couramment utilisés mais trop peu de campagnes sur la Mauldre ont été menées pour pouvoir ne serait-ce que faire un état des lieux. Cet indice nécessite pour son élaboration la présence d'habitats diversifiés, qui font souvent défaut dans des rivières très anthropisées. II reflète la qualité moyenne globale du cours d'eau, ceci nous intéresse peu ici car il nous importe de déceler un type de pollution, principalement organique et minérale. II faut donc faire appel à d'autres indices tels que l'IOBS (LAFONT et al., 1998), principalement basé sur les populations d'oligochètes, qui est un bon descripteur de la pollution organique des eaux et de la contamination des sédiments en métaux lourds. Les indices diatomiques reflètent les impacts ponctuels de pollution car les diatomées sont des organismes à cycles vitaux très courts. Ils sont donc susceptibles d'intégrer des changements instantanés de la qualité des eaux (l'indice IDCR (LAFONT et al., 1998) par exemple reflète bien des teneurs en orthophosphates). Ces indices décrivent plus globalement les pollutions que les indices sur invertébrés et surtout ils témoignent essentiellement de la qualité de l'eau et sont applicables dans tous les milieux, surtout si l'on effectue les prélèvements sur des supports artificiels. Pour compléter les valeurs données par ces indices et affiner les résultats, il est possible de décrire chaque espèce de diatomée trouvée dans le prélèvement pour en savoir plus sur l'écologie du milieu. Les indices diatomées restent encore peu utilisés en France car il y a peu de biologistes capables de déterminer de manière fiable ces espèces. Ils pourraient néanmoins être utilisés sur la Mauldre pour caractériser cette eau de manière globale, en utilisant des supports artificiels, si les groupes de pressions associatifs obtiennent gain de cause auprès des gestionnaires. Cet indicateur biologique viendrait préciser l'indice de stress démographique utilisé dans cet article et qui ne donne qu'un ordre de grandeur de l'anthropisation des rivières.

\section{REMERCIEMENTS}

Nous tenons à remercier vivement toutes les personnes qui nous ont apporté leur aide pour la réalisation de cette étude puis de cet article, et tout particulièrement Guy Bediot, Edith Crouzet et Olivier Blatrix de l'Agence de l'Eau Seine-Normandie pour leurs conseils, ainsi que les techniciens du Laboratoire de Géologie Appliquée de l'Université Paris VI pour les analyses physicochimiques de la campagne de mesures du 17 septembre 1998 sur le ru de Gally. 


\section{RÉFÉRENCES BIBLIOGRAPHIQUES}

AESN, 1991. Bases de définition d'un systèrne d'évaluation de la qualité des cours d'eau : Étude bibliographique. Nanterre, $99 \mathrm{p}$.

DIREN Ile-de-France, 1994. Rivières d'llede-France notre patrimoine, Richesse ou banalisation des milieux aquatiques, Étude de la qualité 1988-1993. DIREN, Cachan.

DIREN Ile-de-France, 1996. Assainissement collectif en lle-de-France (cartes).

EVEN S., 1995. Modélisation d'un écosystème fluvial : la Seine. Le Modèle Prose. Th. École des Mines, 500 p.

INSEE, 1990. Population communale des Yvelines.

LAFONT M. et al, 1988. Comparaison de quatre indices biologiques pour apprécier l'impact de la pollution dans des cours d'eau français. Naturaliste Can., $115,77-87$.

LANGHADE D., 1997. Les rejets des petites stations d'épuration et la qualité des cours d'eau. IAURIF, Paris.

LESOUEF A., ANDRE A., 1982. Mise au point d'un modèle de qualité de la Seine de Montereau à Poses. Comptes rendus $X V I^{e}$ Journées de l'hydraulique : "L'as- sainissement de demain ". S.H.F, Nantes. 7 p.

MEYBECK M. (Ed. Scientif.), 1998b. La Seine en son bassin : fonctionnement écologique d'un écosystème fluvial anthropisé. Elsevier, Paris.

MEYBECK M., 1998a. Man and river interface : multiple impacts on water and particulates chemistry illustrates in the Seine river basin. Hydrobiologia (sous presse).

MORIZE J., 1984. Analyse des bassins versants des principaux affluents de la Seine dans les Yvelines en relation avec les objectifs "Seine propre » en l'an 2000 et la mise à jour de la carte "Épuration des eaux usées " en lle-de-France. IAURIF, Paris.

ROSNAY J.(de), 1975. Le macroscope : Vers une vision globale. SEUIL, Paris.

SATESE 78, 1995. Rapport d'activité : Bilan technique : Fiches techniques des stations d'épuration du département (communales, privées et industrielles).

WOLFF E., 1994. Éléments pour la prise en compte de l'impact des rejets urbains sur les milieux naturels dans la gestion des systèmes d'assainissement, Lyon. Th INSAL, n' $0100,320 \mathrm{p}$. 